\title{
Isolated single lung perfusion with doxorubicin is effective in eradicating soft tissue sarcoma lung metastases in a rat model
}

The only effective therapy for patients with metastatic soft tissue sarcoma in the lung is surgical resection, with a 5-year survival of approximately $25 \%$. Because systemic chemotherapy has not significantly affected survival in these patients, we began to investigate locoregional chemotherapy. We have previously shown that isolated single lung perfusion with doxorubicin in the rat results in higher lung tissue levels and lower systemic toxicity than does high-dose intravenous therapy. In the present study, we examined the safety of isolated lung perfusion with doxorubicin and its efficacy in the treatment of experimental pulmonary metastases from soft tissue sarcoma. In experiment 1, 15 F344 rats were randomized into three groups $(n=5)$ : group I had isolated left lung perfusion with doxorubicin $320 \mu \mathrm{g} / \mathrm{ml}$ in saline solution; group II had left isolated lung perfusion with doxorubicin 480 $\mu \mathrm{g} / \mathrm{ml}$ and group III with doxorubicin $640 \mu \mathrm{g} / \mathrm{ml}$. All perfusions with doxorubicin were at $0.5 \mathrm{ml} / \mathrm{min}$ for 10 minutes followed by perfusion of saline solution for 5 minutes. On day 21, all animals underwent right (contralateral) pneumonectomy and were observed for over 10 days. In experiment 2 , two groups of $F 344$ rats were injected intravenously with $10^{7}$ viable methylcholanthrene-induced sarcoma cells on day 0 . On day 7 , group I $(n=12)$ had left isolated lung perfusion with saline solution only and group II $(n=15)$ had isolated lung perfusion with doxorubicin $320 \mu \mathrm{g} / \mathrm{ml}$. On day 21 , all animals were killed, and their lungs were stained for metastases. Routine histologic sections from three animals from group II were examined. In experiment $1,80 \%$ of the animals in group I survived contralateral pneumonectomy. There were no survivors in groups II and III. In experiment 2, three animals died after isolated lung perfusion (one from group I and two from group II), and one animal (group I) was excluded because of mediastinal tumor. All animals in both groups had massive tumor replacement of the right (untreated) lung. Group I animals had massive tumor replacement of the left (treated) lung, whereas animals in group II had eradication of metastases in nine of ten cases; no microscopic evidence of tumor was detected in all three animals evaluated for microscopic disease. Isolated lung perfusion with doxorubicin $320 \mu \mathrm{g} / \mathrm{ml}$ is safe and effective in eradicating experimental pulmonary sarcoma metastases in this model. (J Thorac Cardiovasc SuRg 1994;107:50-4)

Benny Weksler, MD (by invitation), Jeffrey Lenert, MD (by invitation), Bruce Ng, BS (by invitation), and Michael Burt, MD, PhD, New York, N.Y.

$T_{r e s}$ consists of resection of all pulmonary nodules whenever possible. A 5-year survival of $25 \%$ after complete resec-

From the Thoracic Oncology Laboratory, Thoracic Service, Department of Surgery, Memorial Sloan-Kettering Cancer Center, New York, N.Y.

Read at the Seventy-third Annual Meeting of the American Association for Thoracic Surgery, Chicago, Ill., April 25-28, 1993.

Address for reprints: Michael Burt, MD, PhD, Memorial SloanKettering Cancer Center, 1275 York Ave., New York, NY 10021.

Copyright $(\odot) 1994$ by Mosby-Year Book, Inc.

$0022-5223 / 94 \$ 1.00+.10 \quad \mathbf{1 2} / \mathbf{6} / \mathbf{5 0 4 5 9}$ tion can be expected, as compared with virtually no 5-year survival for those whose disease is not completely resected or inoperable. ${ }^{1-3}$ Adjuvant systemic chemotherapy has not been shown to be beneficial in preventing recurrence or improving survival. ${ }^{4,5}$ Most of the patients in whom therapy fails will die of recurrent neoplastic disease exclusively in the chest. ${ }^{6}$ Recurrence is presumably due to micrometastases present at the time of the initial resection of the metastatic nodules. ${ }^{7}$ Therefore, control of all intrathoracic disease at the initial operation is essential for success.

We have developed a model of in vivo isolated single 


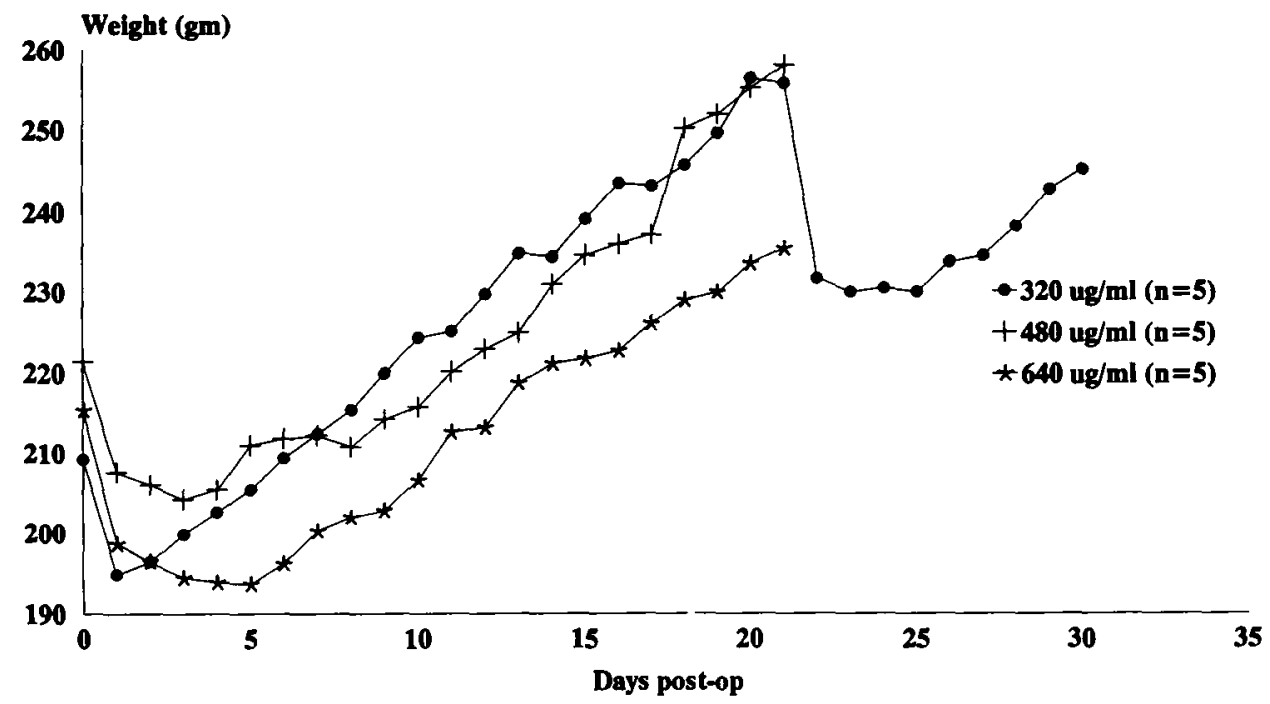

Fig. 1. Daily weight after isolated lung perfusion with doxorubicin. Isolated lung perfusion at day 0 and contralateral pneumonectomy at day 21 . Animals in the groups receiving the higher concentrations of doxorubicin ( 480 and $640 \mu \mathrm{g} / \mathrm{ml}$ ) did not survive contralateral pneumonectomy. post-op, After the operation.

lung perfusion in the rat to study the effects of locoregional therapy. ${ }^{8}$ Isolated single lung perfusion with chemotherapy may be an ideal way to administer high-dose chemotherapy at the time of resection of metastatic nodules with minimal systemic toxicity. The present study was designed to prove the efficacy of isolated single lung perfusion with doxorubicin in the treatment of experimental pulmonary metastases from sarcoma.

\section{Materials and methods}

Male Fischer F344 rats, weighing 200 to 250 gm (Charles Rivers Laboratories, Kingston, N.Y.), were used for all experiments. Animals were treated in compliance with the Animal Welfare Act and the "Principles of Laboratory Animal Care" formulated by the National Society for Medical Research and the "Guide for the Care and Use of Laboratory Animals" prepared by the Institute of Laboratory Animal Resources and published by the National Institutes of Health (NIH Publication No. 86-23, revised 1985). All rats were allowed free access to standard laboratory rat chow (Purina Rat Chow; Ralston Purina Co., St. Louis, Mo.) and water. Housing was temperature controlled and provided a 12-hour light-dark cycle.

Experiment 1. On day 0, 15 animals were randomized into three groups of five animals each. Group I had isolated left lung perfusion with doxorubicin $320 \mu \mathrm{g} / \mathrm{ml}$ in saline solution, group II had isolated left lung perfusion with doxorubicin $480 \mu \mathrm{g} / \mathrm{ml}$ in saline solution, and group III had isolated left lung perfusion with doxorubicin $640 \mu \mathrm{g} / \mathrm{ml}$ in saline solution. Isolated left lung perfusion was performed as previously described. ${ }^{8}$ Animals were anesthetized with pentobarbital $50 \mathrm{mg} / \mathrm{kg}$ intraperitoneally and were intubated orotracheally. Their lungs were ventilated with $100 \%$ oxygen at 70 strokes $/ \mathrm{min}$ with a tidal volume of 10 $\mathrm{mg} / \mathrm{kg}$. Anesthesia was supplemented with $0.5 \%$ halothane as needed. A left thoracotomy was performed through the fourth intercostal space, and the pulmonary artery and vein were dis-

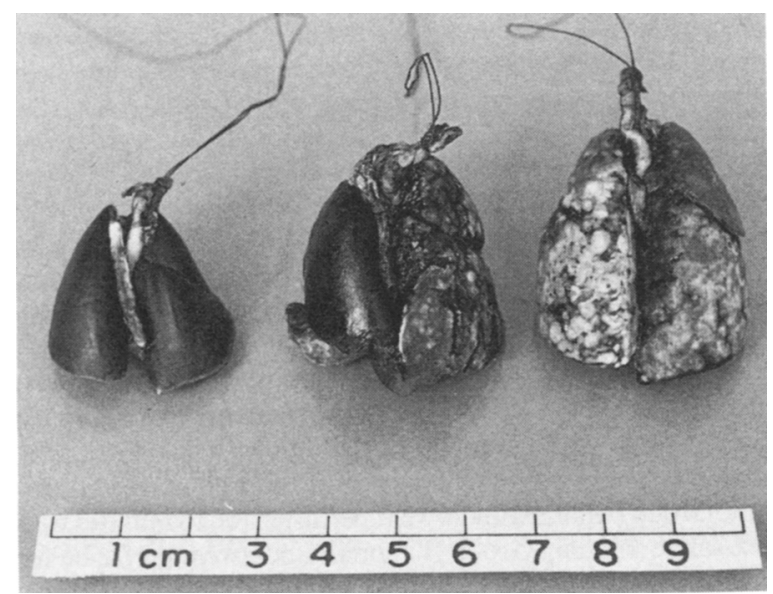

Fig. 2. Posterior view of the lungs of three rats. Left, Normal untreated lungs; middle, lungs after left isolated lung perfusion with doxorubicin; right, lungs after left lung perfusion with saline solution. Normal lung tissue stains are black, and tumor stains are white. After isolated left lung perfusion with doxorubicin, the left lung is cleared of tumor, whereas the right lung is completely infiltrated by metastatic sarcoma. Lung perfusion with saline solution shows no effect.

sected free. With the use of an operating microscope (magnifcation $\times 16$, OpMi-1; Carl Zeiss GmbH, Jena, Germany), a PE-10 catheter (Clay-Adams, Parsippany, N.J.) was placed in the pulmonary artery. The pulmonary artery and vein were clamped proximally, preventing leakage of perfusate into the systemic circulation. The left lung was then perfused for 15 minutes at a rate of $0.5 \mathrm{ml} / \mathrm{min}$, and the pulmonary vein effluent was collected through a venotomy. During the initial 10 minutes, the lung was perfused with doxorubicin and, during the 

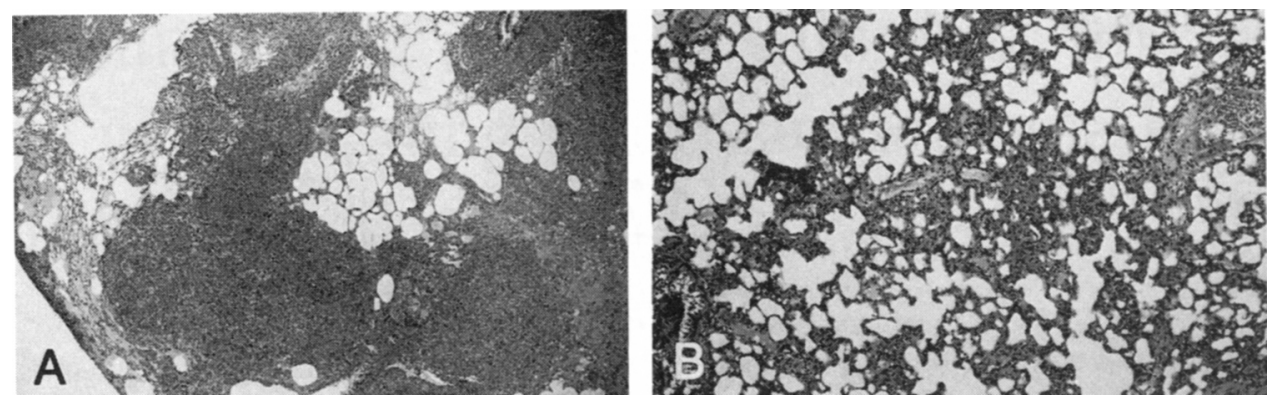

Fig. 3. Histologic characteristics of the lung. A, Left lung after perfusion with doxorubicin; no microscopic tumor is seen. B, Right lung of the same animal completely infiltrated with poorly differentiated sarcoma.

last 5 minutes, with saline solution so excess doxorubicin could be washed out of the pulmonary circulation. At the end of the perfusion, the pulmonary artery and pulmonary vein were repaired with 9-0 nylon suture, and the pulmonary circulation was restored. The chest was closed in three layers, and the animals were extubated. All animals underwent right (contralateral) pneumonectomy on day 21 . Daily weight was recorded after isolated lung perfusion and for 10 days after contralateral pneumonectomy.

Experiment 2. On day 0, all animals had intravenous injections of $10^{7}$ viable methylcholanthrene-induced sarcoma cells via right external jugular vein. Tumor was harvested fresh from a tumor-bearing animal, and single tumor cell suspension was prepared. ${ }^{9}$ This methylcholanthrene-induced sarcoma has been serially passaged subcutaneously in our laboratory and has been extensively characterized. ${ }^{10,11}$ Pulmonary metastases can be reproducibly induced by intravenous injection of single cell suspension. ${ }^{12}$ On day 7 , after tumor inoculation, animals were randomized into two groups: group I animals $(n=12)$ were treated with isolated left lung perfusion with saline solution only. Group II animals $(n=15)$ were treated with isolated left single lung perfusion with doxorubicin $320 \mu \mathrm{g} / \mathrm{ml}$ in saline solution. Isolated left lung perfusion was performed as described previously. Group I animals underwent perfusion for 15 minutes with $0.9 \%$ saline solution. Group II animals underwent perfusion for 10 minutes with doxorubicin and 5 minutes with $0.9 \%$ saline solution. On day 21 , animals were killed, and their lungs were stained with India ink for identification of metastases by the method of Wexler. ${ }^{13}$ By this method, lungs are stained black with india ink and tumor nodules appear white. Three animals in group II underwent routine hematoxylin and eosin histologic staining of their lungs.

Results were analyzed by Fisher's exact test and analysis of variance whenever appropriate. Significance was defined as $p \leq 0.05$.

\section{Results}

Experiment 1. In group I, four of five animals (80\%) survived contralateral pneumonectomy. All animals in groups II and III died within 24 hours after operation of apparent acute respiratory failure. Daily weight after isolated perfusion is depicted in Fig. 1. After isolated perfusion, animals lost weight for 3 to 4 days and then recov- ered. After contralateral pneumonectomy, animals lost weight but within 4 days began gaining weight. No significant difference in the curves was found with analysis of variance.

Experiment 2. Three of 27 animals died immediately after isolated lung perfusion: two from anesthesia complications (one from each group) and one from bleeding (from group II). One animal in group I was excluded at the time of operation because of tumor in the mediastinum presumably as a result of extravasation of the single cell suspension at the time of injection. All animals had massive tumor replacement of the right (untreated) lung. All animals in group I (treated with saline solution) had comparable massive tumor involvement of the left lung. Nine of ten animals $(90 \%)$ in group II (isolated lung perfusion with doxorubicin) had eradication of macroscopic tumor from the left lung ( $p \leq 0.001$ versus group I, Fig. 2). One animal in group II had one $0.5 \mathrm{~mm}$ white patch in the left lung that did not meet our criteria for complete clearance. The histologic lung sections taken from three group II rats showed complete histologic clearing of the left lung; the right lung had massive tumor replacement with poorly differentiated sarcoma (Fig. 3).

\section{Discussion}

Effective systemic chemotherapy for soft tissue sarcoma is lacking. Therefore, in patients with metastatic soft tissue sarcoma in the lungs as their only evidence of disease, resection of the pulmonary metastases, if possible, is currently considered standard therapy by many. The goal of pulmonary resection for metastatic soft tissue sarcoma is complete eradication of disease. When complete resection of all pulmonary metastases is accomplished, the 5 -year survival ranges from $25 \%$ to $30 \%$ in most reported series. However, when resection is not complete, most patients die within 3 years. ${ }^{1-3,6,14}$ Recurrence of disease after complete resection is usually confined to the lungs, ${ }^{6}$ 
making control of pulmonary disease at operation imperative for success. Micrometastases present at the time of the initial operation are the likely cause of recurrent disease. ${ }^{7}$ Doxorubicin is the most effective chemotherapic agent in the treatment of sarcoma ${ }^{15}$ but has not affected survival in most patients with metastatic sarcoma to the lungs. ${ }^{4}$ Systemic doxorubicin administration is associated with a prevalence of dose-limiting side effects, mainly cardiomyopathy, ${ }^{5}$ which precludes high-dose, effective chemotherapy for the treatment of metastatic sarcoma of the lung. We have been studying an animal model of isolated single lung perfusion with doxorubicin to administer locally effective doses of chemotherapy while minimizing potential systemic side effects. We have previously shown that animals treated with isolated single lung perfusion have a 10-fold increase in lung concentration and 10-fold decrease in heart concentration of doxorubicin compared with animals treated by systemic (intravenous) administration. Furthermore, the animals tolerated locoregional administration of doxorubicin much better than they did systemic treatment, maintaining normal growth after isolated lung perfusion, whereas animals that received systemic injections of doxorubicin failed to gain weight. ${ }^{16}$

In the present study, we demonstrated that isolated single lung perfusion with doxorubicin $(320 \mu \mathrm{g} / \mathrm{ml})$ is safe and effective in the treatment of experimental sarcoma lung metastases. Normal animals that underwent perfusion with this dosage were able to tolerate contralateral pneumonectomy, demonstrating tolerance of the perfused lung to doxorubicin. In experiment 2, we replicated a clinical setting of micrometastatic lung disease. Seven days after intravenous injection of sarcoma cells, macroscopic pulmonary nodules cannot be appreciated. Macroscopic disease appears approximately 14 days after intravenous injection of tumor. Lungs perfused with doxorubicin had complete clearance of macroscopic and microscopic tumor, whereas lungs perfused with saline solution alone had massive tumor replacement, demonstrating the efficacy of isolated lung perfusion with doxorubicin in the treatment of sarcoma metastases in this model.

Although other investigators have demonstrated the ability to safely perform isolated lung perfusion with doxorubicin in dogs, ${ }^{17-21}$ the lack of a transplantable tumor did not allow demonstration of the efficacy in treating metastatic tumors in the lung. This article demonstrates, we believe for the first time, that isolated lung perfusion with antineoplastic therapy is effective in eradicating pulmonary metastases, at least in this rat soft tissue sarcoma model. We strongly believe that this technique for administering antineoplastic therapy holds promise in the treatment of patients with pulmonary metastases.

\section{REFERENCES}

1. Casson AG, Putnam JB, Natarajan G, et al. Five-year survival after pulmonary metastasectomy for adult soft tissue sarcoma. Cancer 1992;69:662-8.

2. Martini N, McCormack PM, Bains MS, Beattie EJJ. Surgery for solitary and multiple pulmonary metastases. N Y State J Med 1978;78:1711-3.

3. McCormack PM, Bains MS, Beattie EJ, Martini N. Pulmonary resection in metastatic carcinoma. Chest 1978; 73:163-6.

4. Lanza LA, Putnam JB Jr, Benjamin RS, Roth JA. Response to chemotherapy does not predict survival after resection of sarcomatous pulmonary metastases. Ann Thorac Surg 1991;51:219-24.

5. Casper ES, Gaynor JJ, Hajdu SI, et al. A prospective randomized trial of adjuvant chemotherapy with bolus versus continuous infusion of doxorubicin in patients with highgrade extremity soft tissue sarcoma and an analysis of prognostic factors. Cancer 1991;68:1221-9.

6. Potter DA, Glenn J, Kinsella T. Patterns of recurrence in patients with high grade soft-tissue sarcomas. J Clin Oncol 1985;3:353-66.

7. Huth JF, Holmes EC, Vernon SE, Callery CD, Ramming KP, Morton DL. Pulmonary resection for metastatic sarcoma. Am J Surg 1980;140:9-16.

8. Weksler B, Schneider A, Ng B, Burt ME. Isolated single lung perfusion in the rat: an experimental model. J Appl Physiol 1993;74:2736-9.

9. Madden RE, Burk D. Production of viable single cell suspension from solid tumors. J Nat Cancer Inst 1961;27:84161.

10. Burt ME, Lowry SF, Gorschboth CM, Brennan MF. Metabolic alteration in a noncachectic animal tumor system. Cancer 1981;47:2138-46.

11. Popp MB, Morrison SD, Brennan MF. Total parenteral nutrition in a methylcholanthrene-induced rat sarcoma model. Cancer Treat Rep 1981;65:137-43.

12. Nagashima A, Yasumoto K, Nakahashi H, Furukawa T, Inokuchi K, Nomoto K. Establishment and characterization of high and low-metastatic clones derived from a methylcholanthrene-induced rat fibrosarcoma. Cancer Res 1986;46:4420-4.

13. Wexler H. Accurate identification of experimental pulmonary metastasis. J Nat Cancer Inst 1966;36:641-5.

14. Jablons D, Steinberg SM, Roth J, Pittaluga S, Rosenberg SA, Pass HI. Metastasectomy for soft tissue sarcoma: further evidence for efficacy and prognostic indicators. J THORAC CARdiovasC SuRG 1989;97:695-705.

15. Gottlieb JA, Baker LH, O'Bryan RM, et al. Adriamycin (NSC-123127) used alone and in combination for soft tissue and bony sarcoma. Cancer Chemother Rep 1975; 6:271-82.

16. Weksler B, Ng B, Lenert J, Burt M. Isolated single lung 
perfusion with doxorubicin is pharmacokinetically superior to systemic administration. Ann Thorac Surg 1993;56:20914.

17. Minchin RF, Johnston MR, Schuller HM, Aiken MA, Boyd MR. Pulmonary toxicity of doxorubicin administered by in situ isolated lung perfusion in dogs. Cancer 1988;61:1320-5.

18. Minchin RF, Johnston MR, Aiken MA, Boyd MR. Pharmacokinetics of doxorubicin in isolated lungs of dogs and humans perfused in vivo. J Pharmacol Exp Ther 1984; 229:193-8.

19. Johnston MR, Minchin RF, Shull JH, et al. Isolated lung perfusion with adriamycin: a preclinical study. Cancer 1983;52:404-9.

20. Johnston MR, Christensen CW, Minchin RF, et al. Isolated total lung perfusion as a means to deliver organ-specific chemotherapy: long-term studies in animals. Surgery 1985; 98:35-44.

21. Baciewicz FA, Arredondo M, Chaudhuri B, et al. Pharmacokinetics and toxicity of isolated perfusion of lung with doxorubicin. J Surg Res 1991;50:124-8.

\section{Discussion}

Dr. Harvey I. Pass (Bethesda, Md.). My congratulations to Drs. Weksler and Burt for an appropriate extension of some of the work performed by Dr. Michael Johnson at the National Cancer Institute in the early 1980s. Presently at our institution, we are using isolated lung perfusion but not with Adriamycin. We are using interferon and tumor necrosis factor in human isolated lung perfusions as a Phase I trial.
Is this model the same that would be used in your human studies of Adriamycin; in other words, how long would you perfuse the lungs? Do you think that you should perfuse longer or shorter than the usual 90 minutes? Can you tell us of the longterm results in the animals that have less metastases and whose lungs have been perfused? In other words, if the animals are not killed, how many animals are subsequently cured with the single perfusion with Adriamycin?

Dr. Douglas J. Mathisen (Boston, Mass.). Dr. Weksler, could you also comment on any histologic evidence of injury to the perfused lung that you may have observed?

Dr. Weksler. In answer to Dr. Pass's questions, I believe that we are not ready yet to perfuse human lungs in this manner. We are currently evaluating the relation of the perfusion rate and time and the perfusate concentration to final lung levels of doxorubicin. It is possible that by manipulating the rate and concentration we will be able to perfuse for shorter periods of time without compromising the final lung levels of the drug. The long-term results in the rat are hard to evaluate. Although we have histologic clearing of the lung perfused with doxorubicin, the contralateral lung is not treated, and animals eventually die of tumor in the right (untreated) lung.

Dr. Mathisen, I do have results from histologic studies in normal lungs perfused with doxorubicin. After perfusion with doxorubicin $320 \mu \mathrm{g} / \mathrm{ml}$, the lung looks fairly normal except for mild pleural thickening. After perfusion with doxorubicin 640 $\mu \mathrm{g} / \mathrm{ml}$, the lung is severely injured, with obliteration of air spaces and fibrosis. We also looked at the histologic characteristics after perfusion of a lung containing tumor with doxorubicin 320 $\mu \mathrm{g} / \mathrm{ml}$. Although no tumor can be visualized, a moderate amount of interstitial fibrosis is observed. 